\title{
Novel experimental techniques for neutron induced charge particle reaction studies in nuclear astrophysics
}

\author{
Chandrabhan Yadav $^{1, *}$ and Moshe Friedman ${ }^{1, * *}$ \\ ${ }^{1}$ Racah Institute of Physics, Hebrew University of Jerusalem, 91904 Jerusalem, Israel
}

\begin{abstract}
Neutron induced reaction studies on various unstable and short-lived nuclei play a very important role in understanding the various nucleosynthesis processes occuring in astrophysics scenario. We discuss here our approach to study neutron induced charge particle cross-section for various unstable and short-lived nuclei at effective temperatures of $\sim 1.5-3.5 \mathrm{GK}$ using the ${ }^{7} \mathrm{Li}(p, n)$ reaction as a neutron source with five orders of magnitude higher neutron intensities with respect to currently available neutron time-of-flight facilities, using SARAF accelarator and Liquid Lithium Target (LiLiT) facility.
\end{abstract}

\section{Introduction}

Measurements of neutron-induced reaction rates on various unstable and short-lived nuclei are imperative for a complete understanding of the nucleosynthesis occurring in various astrophysical sites and events. The neutron time-of-flight (TOF) method is the most straightforward approach to carry out such experiments. TOF facilities use long flight distance typically few tens of meters and above, resulting in dramatic reduction in neutron intensity. The CERN n_TOF facility EAR2, currently the most intense neutron TOF facility, provide $10^{6}$ neutrons/second at relevant energy range for astrophysics studies [1]. An alternative approach suggested in Ref [2], extensively used for the last three decades. They suggested to use the ${ }^{7} \mathrm{Li}(p, n)$ reaction as a neutron source with a specific proton energy at $1.912 \mathrm{MeV}$, close to reaction threshold at $1.88 \mathrm{MeV}$ and a thick Li target. At this proton energy, the neutrons are forward-collimated and their energy distribution resembles Maxwellian flux distribution with a temperature of $\approx 0.3 \mathrm{GK}$, allowing nearly direct measurement of the Maxwellian Averaged Cross-Section (MACS) at $0.3 \mathrm{GK}$, relevant for some s-process sites [3]. An important advantage of this approach is substantial increase in the neutron intensity up to $\approx 10^{9}$ neutrons/second for $100 \mu \mathrm{A}$ proton beam. The intense proton beam from superconducting linear RF accelarator SARAF provide beam current of few $\mathrm{mA}$ and thus allowing measurements with even higher neutron intensity on the order of $10^{10}$ neutrons/second $[4,5]$. The four order of magnitude increase in neutron intensity allows measurement of smaller crosssection reactions and short-lived isotope. In spite of the successful use of ${ }^{7} \operatorname{Li}(p, n)$ reaction for such experiments, it is only limited for a specific temperature of $\approx 0.3 \mathrm{GK}$. Various suggestions [6-8] were made to obtain neutron fields at higher temperatures. In this work we discuss our approach to extend the use of varying proton energies in the range of 1.9-3.6 $\mathrm{MeV}$ on thick lithium targets to carry out neutron-induced cross sections measurements in

\footnotetext{
*e-mail: chandrabhan.yadav@mail.huji.ac.il

**e-mail: moshe.friedman@mail.huji.ac.il
} 
the temperatures region of 1.5-3.5 GK. The higher proton energies will result with neutron intensities on the order of $10^{11}$ neutrons/sec on the sample. Nucleosynthesis in explosive scenarios does not occur in a specific temperature, thus it is important to determine MACS values continuously through-out this temperature region. Our proposed method is based on a series of cross section measurements at different proton energies with proper weighting to obtain the MACS values at any given temperature in this range. The method to produce the neutron field, MACS values at different temperature and required computation tool is described in detail in a recent publication [9]. We will use this method to study $(n, p)$ and ( $n$, $\alpha$ ) cross-sections at explosive stellar temperatures, for various nuclei for which experimental data is poor or even non-existent.

\section{Experimental plans and details}

We will carry out our $(n, p)$ and $(n, \alpha)$ cross-sections measurements for various unstable and short-lived nuclei in two-phases. Figure 1 shows schematic of conceptual design of experimental set-up for Phase-I and Phase-II experiments and respective detection set-up. In Phase-I, experiments with stable and long-lived nuclei will be done at a low intensity proton accelerator. As illustrated in left panel of Fig. 1, a proton beam with varying energies at 1.9 3.6 MeV impinges on a thick Li target to produce neutron beam which further impinges on a sample place inside the micromegas based gaseous detector located $2 \mathrm{~cm}$ downstream to the neutron source. The reactions product will be detected by the gas filled detector. Phase-II of the project dedicted to experiments with short-lived nuclei will make use of full intensity of SARAF, above $1 \mathrm{~mA}$ with Liquid Lithium Target (LiLiT) as a neutron source. As illustrated in right panel of Fig. 1, a proton beam with varying energies impinges on $1.5 \mathrm{~mm}$ thick liquid lithium target. The sample is located $1 \mathrm{~cm}$ downstream the neutron source. The outgoing charge particles are directed using a quadruple and a dipole magnets to be detected at focal plane nearly $3.5 \mathrm{~m}$ from the neutron source. We plan to carry out for example, ${ }^{26} \mathrm{Al}(n, p)$, ${ }^{26} \mathrm{Al}(n, \alpha)\left[\mathrm{t}_{1 / 2}=7.2 \times 10^{5}\right.$ Years $],{ }^{40} \mathrm{~K}(n, p),{ }^{40} \mathrm{~K}(n, \alpha)\left[\mathrm{t}_{1 / 2}=1.25 \times 10^{9}\right.$ Years] and ${ }^{56} \mathrm{Ni}(n, p)$ $\left[\mathrm{t}_{1 / 2}=6.08\right.$ Days $],{ }^{75} \mathrm{Se}(n, p)\left[\mathrm{t}_{1 / 2}=119.8\right.$ Days $]$ reactions with Phase-I and Phase-II set-up respectively.

We are currently pursuing Phase-I experiments and developing a micromegas based gaseous detector. The detector will be made in cylindrical geometry with cathode, equipotential rings and micro pattern gas amplifier i.e., micromegas structure stretched over the segmented anode pads. The sample for nuclear reaction of interests will be placed in the center of the cathode inside the chamber facing the anode plane. Such design of detection system will provide solid angle of $2 \pi$ for particle detection. The separation between cathode and anode plane will be $18 \mathrm{~cm}$, and the active diameter of cathode and anode will be around $14 \mathrm{~cm}$. The anode plane will be divided into five circular rings with central pad ring of diameter $3 \mathrm{~cm}$ and second, third and fourth rings of diameter 6,9 and $12 \mathrm{~cm}$ respectively, and fifth ring of diameter $14 \mathrm{~cm}$. These outer rings are further segmented into 8 section thus giving total of 33 segmented pads on the anode plane. Left panel of Fig. 2 shows segmented anode pad as designed in Geant4 [10] simulation. The gas mixture of $\mathrm{Ne}(90 \%)$ and $\mathrm{CF}_{4}(10 \%)$ in the pressure range of $3-4 \mathrm{~atm}$ will be used as a detection gas. Fig. 2 (right panel) shows electron drift velocity as a function of field for various gas mixtures obtained using [11]. The $\mathrm{Ne} / \mathrm{CF}_{4}$ gas mixture is found to be better choice as detection gas as mixtures containing argon and hydrogen had to omitted in order to avoid background from neutron induced reaction with $\mathrm{Ar}$ and $\mathrm{H}$. Geant 4 simulation of the detector shows that detector can be operated with proton beam current of $10 \mu \mathrm{A}$ in a strong $\gamma$ radiation environment from ${ }^{7} \operatorname{Li}\left(p, p^{\prime} \gamma\right)$ and neutron background. 


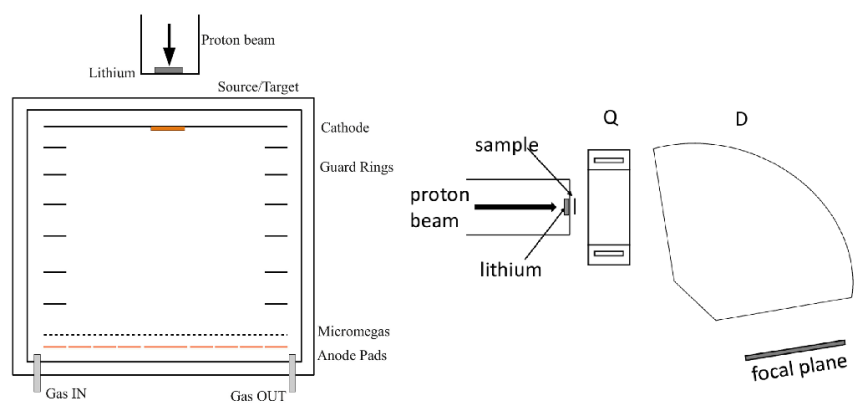

Figure 1. (Left) Schematic of conceptual experimental set-up for Phase-I experiments, and micromegas based gaseous detector. (Right) Schematic of conceptual experimental set-up for Phase-II experiments, and large angle spectrometer.
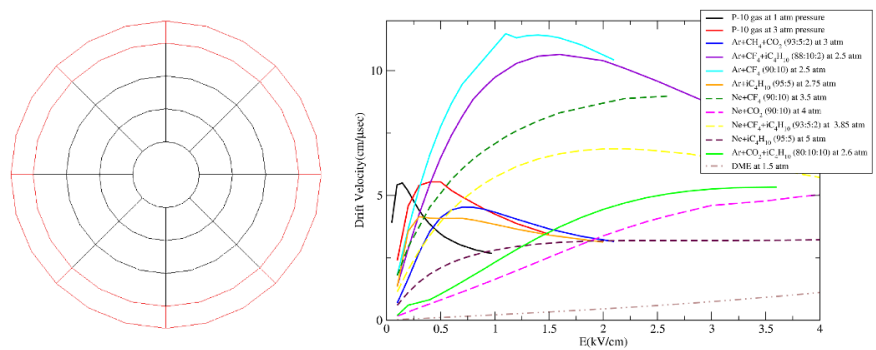

Figure 2. (Left) Segmented anode pad as designed in Geant4 simulation. (Right) Electron drift velocity as a function of field for various gas mixtures obtained using [11].

\section{Summary and outlook}

Measurements of neutron-induced cross sections on various nuclei of astrophysical interests at explosive stellar temperatures is being plan to study with high-intensity neutron fluxes at the relevant energies. We plan to carry out our first experiments for ${ }^{26} \mathrm{Al}(n, p)$ and ${ }^{26} \mathrm{Al}(n, \alpha)$ reactions with a gas detector being designed and developed very soon.

\section{References}

[1] C. Weiß et al., Nucl. Instrum. Method A 799, 0168-9002 (2015).

[2] W. Ratynski, and F. Käppeler, Phys. Rev. C 37, 595 (1988).

[3] F. Käppeler, R. Gallino, S. Bisterzo, W. Aoki, Rev. Mod. Phys.83, 157 (2011).

[4] I. Mardor, O. Aviv, M. Avrigeanu et al., Eur. Phys. J. A 54, 91 (2018).

[5] M. Paul et al., Eur. Phys. J. A 55, 44 (2019).

[6] M. Heil et al., Phys. Rev. C 71, 025803 (2005).

[7] R. Reifarth et al., Eur. Phys. J. Plus 133, 424 (2018).

[8] R. Reifarth et al., Phys. Rev. C 77, 015804 (2008).

[9] M. Friedman, Eur. Phys. J. A. 56, 155 (2020).

[10] S. Agostinelli et al., Nucl. Inst. Methods Phys. Res. A 506, 250-303 (2003).

[11] Garfield++, https://garfieldpp.web.cern.ch/garfieldpp/ 